Relations industrielles

Industrial Relations

Some Theories or Organization, Revised Edition, by Albert $\mathrm{H}$. Rubenstein and Chadwick J. Haberstroh, Richard D. Irwin, Inc., Homewood, Illinois, 1966, 722 pages.

\title{
Paul Bouchard
}

Volume 22, numéro 1, 1967

URI : https://id.erudit.org/iderudit/027770ar

DOI : https://doi.org/10.7202/027770ar

Aller au sommaire du numéro

Éditeur(s)

Département des relations industrielles de l'Université Laval

ISSN

0034-379X (imprimé)

1703-8138 (numérique)

Découvrir la revue

Citer ce compte rendu

Bouchard, P. (1967). Compte rendu de [Some Theories or Organization, Revised Edition, by Albert H. Rubenstein and Chadwick J. Haberstroh, Richard D. Irwin, Inc., Homewood, Illinois, 1966, 722 pages.] Relations industrielles / Industrial Relations, 22(1), 140-140. https://doi.org/10.7202/027770ar

Tous droits réservés @ Département des relations industrielles de l'Université Laval, 1967
Ce document est protégé par la loi sur le droit d'auteur. L'utilisation des services d'Érudit (y compris la reproduction) est assujettie à sa politique d'utilisation que vous pouvez consulter en ligne.

https://apropos.erudit.org/fr/usagers/politique-dutilisation/ 
Cette revue mensuelle contient surtout des textes et des résumés de décisions légoles en ropport ovec les relations potronalesouvrières. Elle veut fournir à tous ceux que concerne l'ospect légol de cette question, employeurs, dirigeants syndicaux, oviseurs légoux, étudiants, - une information soignée et même un instrument de travail. Les éditeurs insistent sur leur volonté de tenir les lecteurs oux faits des dernières nouveautés. Par exemple, l'éditorial nous révèle que l'événement le plus importont des derniers mois dans le domaine couvert par la revue fut la passosion, le 12 ooût 1966 , du * Pieces and Income Act ». Nous savons tous que la décision prise por le gouvernement de $M$. Wilson de geler, pour une période fixe, les solaires et les prix o fait bien du bruit dans le monde.

Knight's Industrial Reports nous fournit le texte complet d'un mémoire intitulé: «Pieces and Income Standstill », qui fut présenté ou parlement par le Secrétaire d'Etot en juillet 1966 et qui nous permet de saisir très bien le sens, le fonctionnement et les implications de cette loi.

Comme outres services, lo revue fournit, entre autres, une liste des décisions des tribunaux d'arbitrage, résume sous forme de cas des décisions de la Cour d'appel et plusieurs autres décisions gouvernementales clossées selon les ministères.

\section{André PETIT}

Some Theories of Organization, Revised Edition, by Albert $H$. Rubenstein and Chadwick J. Haberstroh, Richard D. Irwin, Inc., Homewood, Illinois, 1966, 722 poges.

$\mathrm{Ce}$ volumineux ouvrage que nous présente les deux auteurs est une édition améliorée du volume qui avait été publié en 1960 par les mêmes auteurs. Des progrès incessants dans les théories de l'organisation associés cux développements rapides qui se sont produits dans les sciences du comportement ont rendu nécessaire lo recherche et l'étude de nouvelles opportunités.

Cette seconde édition ne se veut pas un document scientifique très rigoureux. Elle est centrée, cependant, sur le concept de la théorie organisationnelle en tant qu'elle est une approche à une étude systématique du comportement à l'intérieur d'une organisation. Les articles qui sont consignés dans ce volume se devraient de familiariser le lecteur avec une variété d'approches conceptuelles et méthodiques toujours empreintes de réalisme et d'opérationalité. Les auteurs espèrent également favoriser d'une façon spécifique une meilleure compréhension de la période transitoire qui se situe entre les sciences sociales à l'état pur et l'amélioration des pratiques, techniques et méthodes directoriales.

Si nous regardons de plus près le contenu du volume, nous constatons qu'une première partie traite de la nature des théories de l'organisation et on voit apparaitre alors des noms comme Homans et Lewin. Lo deuxième partie, de son côté, traite de la structure et du processus organisationnels et on retrouve alors des textes de Weber, Udy, Selznick, Barnard et Dalton. Une troisième partie porte sur le leadership et le moral avec des auteurs tels que: Argyris, Crozier et Hutchins. Une quatrième partie traite de la planification des changements dans les attitudes, les structures et la technologie. On y traite ensuite d'une façon successive des communications, du contrôle et de l'évaluation de l'efficacité et de la performance, du processus de prise de décision et de certaines études techniques.

Ce volume est réellement susceptible $d^{\prime}$ intéresser bien des gens au plus hout point. C'est pourquoi, ceux qui voudraient se familiariser davantage avec les théories administratives ou encore ceux qui oimeraient consulter un bon volume de références en la matière auraient tout avantage à aller puiser chez lui les renseignements dont ils ont besoin.

\section{Paul BOUCHARD}

Sky Full of Storm: A Brief History of California Labor, by David F. Selvin, Center for Labor Research and Education, Institute of Industrial Relations, University of California, Berkeley. 1966.86 pages.

This publication is a brief history of $\mathrm{Ca}$ lifornia labor. One thing quite interesting obout this book is the reason of its appearance on the market. It seems, as Mr. Vial noted it in the foreword, that the author decided to publish such a work to fill a the lock of readily available information on the history of Colifornia labor $\$$.

Sky Full of Storm makes no pretense at being an history in the traditional sense. It develops a feeling for labor struggles in $\mathrm{Ca}$ lifornia and depicts some of the pressing issues and problems confronting organized labor today. These pages deal with fragments of a hundred and fifteen years of $\mathrm{Ca}$ lifornia history - a dozen or so episodes that seemed to Mr Selvin to characterize the 